\title{
Images
}

\section{ICD Lead Dislodgement and Inappropriate ICD Shocks}

\author{
Pablo Blanco, MD*
}

\section{ICD LEAD DISLODGEMENT AND INAPPROPRIATE ICD SHOCKS}

A 16-year-old girl with a history of peripartum cardiomyopathy and implantation of a cardioverter- defibrillator (ICD) ten days before was admitted to the emergency department (ED) because she experienced at least seven episodes of ICD shocks over the last two hours. The ICD generator was noted in the
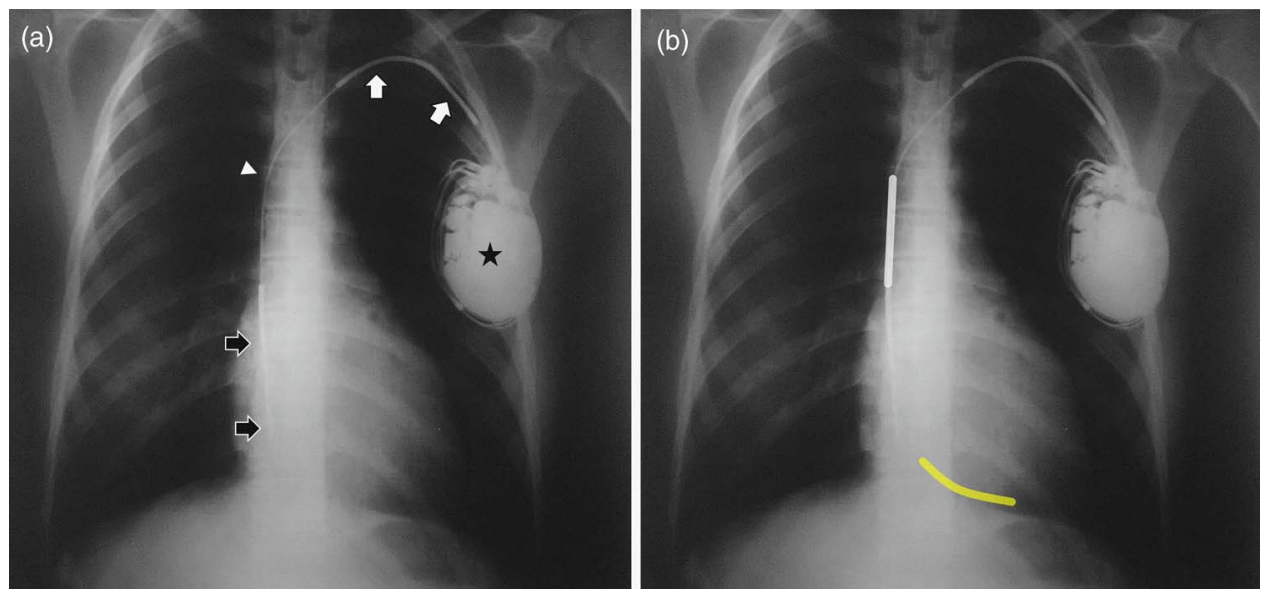

Figure 1. (a) Chest radiograph showing the ICD generator (black star), ICD lead (arrowhead), and the two coils (arrows) for providing the electric shock. The superior vena cava coil (white arrows) is displaced into the left subclavian vein and the right ventricular lead (black arrows) is displaced into the right atrium, directed toward the region of the inferior vena cava (IVC).

(b) Same chest radiograph of (a) indicating the proper position of the shock coils into the superior vena cava (white line) and right ventricle (yellow line).
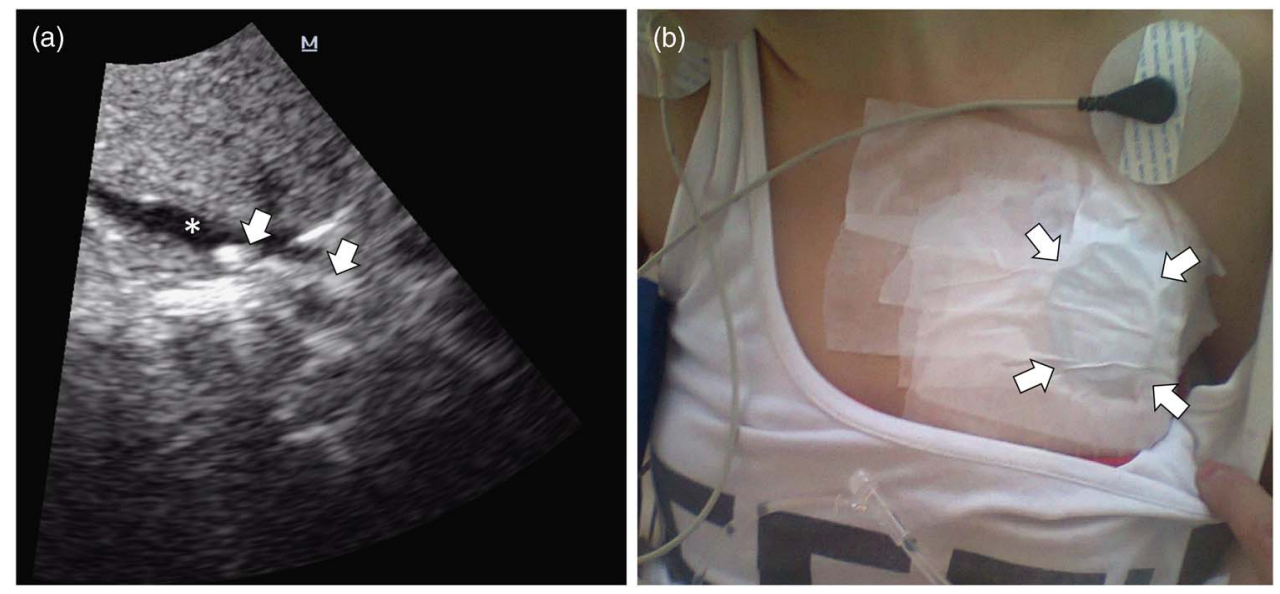

Figure 2. (a) Transthoracic echocardiogram demonstrating the tip of the ICD lead and RV coil (white arrows) entering into the IVC (asterisk). (b) A magnet (white arrows) is placed over the ICD generator and is secured with tape to maintain the inactivated status.

From the *Intensive Care Unit, Hospital Dr. Emilio Ferreyra, Necochea, Argentina.

Correspondence to: Pablo Blanco, Intensive Care Unit, Hospital Dr. Emilio Ferreyra, 4801, 59 St., Necochea 7630, Argentina; Email: ohtusabes@ gmail.com 
left infraclavicular region, with some tenderness on palpation. A normal sinus rhythm was recorded on the surface electrocardiogram. A subsequent witnessed ICD shock was not related to any arrhythmia detected on cardiac monitor. A chest radiograph demonstrated a single-chamber ICD and lead dislodgement, with the resultant misplacement of both ICD shock coils (Figure 1). Transthoracic echocardiogram also confirmed the lead displaced into the inferior vena cava (Figure 2a). A magnet was applied over the ICD generator and device firing was abolished (Figure 2b).

Inappropriate or unnecessary ICD shocks commonly occur as a result of changes in device sensing due to alterations in lead integrity, improper lead position (as in this case) and supraventricular tachyarrhythmias. ${ }^{1,2}$ In order to eliminate this life-threatening ICD complication, a magnet should be positioned over the device to temporarily suspend tachyarrhythmia intervention until ICD problems are identified and resolved, ${ }^{1,2}$ and thus it should be required to have a magnet available in all EDs.

Competing interests: None to declare.

Keywords: Implantable Cardioverter-Defibrillators, Diagnostic X-Ray, Echocardiography, Magnet

\section{REFERENCES}

1. Somani R, DeJong P, Michael K, et al. Unnecessary shock from an implantable cardioverter-defibrillator following transcutaneous pacing. CFEM 2014;16(4):330-3.

2. Pinski SL. Emergencies related to implantable cardioverterdefibrillators. Crit Care Med 2000;28(10 Suppl):N174-80. 